\title{
In Vivo Measurement of Phenylalanine in Human Brain by Proton Nuclear Magnetic Resonance Spectroscopy
}

\author{
EDWARD J. NOVOTNY, JR., MALCOLM J. AVISON, NORBERT HERSCHKOWITZ, \\ OGNEN A. C. PETROFF, JAMES W. PRICHARD, MARGRETTA R. SEASHORE, AND \\ DOUGLAS L. ROTHMAN \\ Departments of Pediatrics [E.J.N., M.R.S.], Human Genetics [M.R.S.], Neurology \\ [E.J.N., O.A.C.P., J.W.P.], and Internal Medicine [M.J.A., D.L.R.], School of Medicine, Yale University, \\ New Haven, Connecticut 06510, and Department of Pediatrics, University of Berne, \\ Berne, Switzerland [N.H.]
}

\begin{abstract}
Disorders of the CNS are the major causes of morbidity and mortality observed in untreated subjects with phenylketonuria (PKU). A method to measure cerebral concentrations of phenylalanine (Phe) in vivo would greatly enhance the ability to investigate both the pathophysiology and the efficacy of therapy of this aminoacidopathy. Twelve image-guided localized proton nuclear magnetic resonance spectroscopic studies were performed in seven subjects with PKU using pulse sequences optimized to detect the aromatic protons of Phe. Ten control studies were also performed using a 2.1-Tesla Bruker Biospec spectrometer. Plasma Phe was measured at the time of the spectroscopic examination in the PKU patients. A Phe signal was observed in all 12 studies performed on the group with PKU, and in five studies cerebral Phe concentrations were measured to be 480 to $780 \mu \mathrm{mol} / \mathrm{g}$. Plasma Phe concentrations were 0.7 to $3.3 \mathrm{mM}$ $(10.8$ to $54.8 \mathrm{mg} / \mathrm{dL})$ in the subjects with PKU. Human cerebral Phe concentrations can be measured noninvasively using proton nuclear magnetic resonance spectroscopy. A simultaneous measure of Phe and several other cerebral metabolites is obtained with this innovative technology. Adaptations of this technique can be
\end{abstract}

Hyperphenylalaninemia due to a deficiency of phenylalanine 4-monooxygenase (EC 1.14.16.1) activity is the most common treatable disorder of amino acid metabolism in man (1). Several mutations in the gene for this enzyme mapped to chromosome $12 q$ have been identified as causing this autosomal recessive disorder (2). Recent studies are beginning to deter-

Received for rapid publication September 29, 1994; accepted November 15, 1994. Correspondence and reprint requests: Edward J. Novotny, Jr., M.D., Yale University, School of Medicine, Department of Pediatrics, LMP 3089, 333 Cedar St., New Haven, CT 06510 .

Supported in part by grants from the National Institute of Neurologic Diseases and Stroke (NS 28790, NS 21708, NS 32126), the National Institute of Diabetes, Digestive Diseases and Kidney (DK 34576), and the Swiss National Science Foundation (313090991).

A portion of this work was presented at the 8th annual meeting of the Society of Magnetic Resonance in Medicine in Amsterdam, Netherlands, in August 1989. used to investigate PKU and other neurometabolic disorders with modifications of current clinical magnetic resonance imaging systems. (Pediatr Res 37: 244-249, 1995)

\section{Abbreviations}

Cho, choline-containing compounds

$\mathrm{Cr}$, creatine

${ }^{\mathbf{1}} \mathbf{H}$, proton

$\mathbf{I R}$, inversion recovery

MRI, magnetic resonance imaging

NA, N-acetyl-containing compounds

NMR, nuclear magnetic resonance

Phe, phenylalanine

PKU, phenylketonuria

ppm, parts per million

TMA, trimethylamine-containing compounds

T1, spin-lattice relaxation time

TE, total echo time

TR, repetition time mine the molecular basis for the phenotypic heterogeneity of this disorder $(3,4)$. Classic PKU is observed in 1 in 7000 to 1 in 15000 births in most white populations. If Phe intake is not restricted, PKU leads to mental retardation, epilepsy, and other neurologic disorders. The advent of newborn screening has produced an enlarging cohort of young adults for whom there is a need to optimize therapy. Two recognized problems facing these individuals are progressive neuropsychologic deterioration after discontinuation of dietary therapy and increased risk of mental retardation in offspring of affected women resulting from fetal exposure to high maternal blood Phe.

Attempts to correlate blood levels of Phe, its metabolites, or genotype with neurologic complications have been enigmatic $(5,6)$. There has been much controversy regarding the mechanism by which hyperphenylalaninemia results in neurologic 
disturbances $(7,8)$. Discontinuation of dietary therapy can result in increased incidence of neuropsychologic problems $(9$, 10). A link between neurologic status and dietary Phe control is now emerging from recent neuroimaging studies demonstrating abnormalities of white matter on MRI (11-13). However, the pathophysiology and the timing of the development of these white matter changes is unknown. Despite these controversies, several groups have recommended strict adherence to dietary therapy (14). A method for noninvasive measurement of Phe and other metabolites in the human brain would permit better management of these problems, and it would provide a better understanding of the pathogenesis of the neurologic disorders observed in PKU. After investigating an animal model of this disorder using ${ }^{1} \mathrm{H}$ NMR spectroscopy (15), we used this noninvasive technique to investigate humans with this aminoacidopathy. Subjects with classic PKU were investigated by a modification of the NMR technique used in our animal study.

\section{METHODS}

Twelve ${ }^{1} \mathrm{H}$ NMR spectroscopic studies were performed on three male and four female subjects aged 13 to $28 \mathrm{y}$ with classic PKU. Seven studies were performed on five PKU subjects using one NMR pulse sequence, and five studies were performed on four PKU subjects using a second NMR pulse sequence designed to permit quantitation of cerebral Phe. Ten control studies were obtained. Five studies on five control subjects aged 22 to 38 y using the first NMR pulse sequence and five studies on five control subjects aged 24 to $37 \mathrm{y}$ using the second quantitative NMR pulse sequence were also performed. Plasma Phe levels were measured at the time of the spectroscopic study in the subjects with PKU.

NMR spectroscopy. ${ }^{1} \mathrm{H}$ spectra were obtained using an extensively modified Biospec spectrometer (Bruker, Billerica, MA) with a 1-m bore, 2.1-Tesla magnet. For studies using the second pulse sequence (see below), the system was equipped with shielded gradients. A home-built 8 - $\mathrm{cm}$ circular surface coil was used for both radiofrequency transmitting and receiving. An MRI of the head was acquired with the surface coil using an IR sequence (TR $=3 \mathrm{~s}, \mathrm{TE}=40 \mathrm{~ms}, \mathrm{IR}=800 \mathrm{~ms}$ ). The first NMR spectroscopic pulse sequence was designed to optimize water suppression and provide maximum excitation at the aromatic protons of Phe using a semiselective excitation pulse. This study was initially designed to determine whether Phe could be observed and to determine the optimum experimental parameters that would be required for quantitation of the Phe signal. A second NMR pulse sequence was then designed to quantitate cerebral Phe concentration. Spectra were acquired from a $15-$ to $32-\mathrm{mL}$ volume using one of the following pulse sequences.

In the first sequence with which initial studies were performed, the volume of interest was localized in the left hemisphere centered 3 to $4 \mathrm{~cm}$ deep from the scalp in the temporoparietal region using the image selected in vivo spectroscopy (ISIS) localization technique (16). Surface suppression was achieved using both sinc and depth pulses fol- lowed by dephasing gradients. Semiselective excitation and refocusing pulses with the maximum excitation centered at the aromatic protons of Phe at $7.3 \mathrm{ppm}$ and a spin echo sequence were used to suppress water. Additionally, either a 2-s presaturation pulse or a modified water eliminated Fourier transform sequence using a 256-point phase-swept hyperbolic secant pulse centered on water was used to enhance the water suppression. TE of 10 to $20 \mathrm{~ms}$ and TR of 3 or $6 \mathrm{~s}$ were used. Shimming was performed using the surface suppression sequence and a nonselective spin echo. One hundred twentyeight free induction decays were acquired for each spectrum in approximately $6 \mathrm{~min}$. Two to four proton spectra were acquired in each study.

In the second sequence, a $15-\mathrm{mL}$ volume was localized in the occipital cortex using image selected in vivo spectroscopy, and selective excitation centered in the midline. Localized automatic shimming was performed on all first- and secondorder shim coils according to a recently published procedure (17). The line widths of the singlets (e.g. total $\mathrm{Cr}$ ) were usually 5 to $7 \mathrm{~Hz}$ after this procedure. Technical details of localization and water suppression are given elsewhere (18). The semiselective pulse delay was chosen to provide maximum excitation at the aromatic protons of Phe at $7.3 \mathrm{ppm}$. The TE time was $15 \mathrm{~ms}$ and the TR was $4.1 \mathrm{~s}$. Two spectra consisting of 128 free induction decays were obtained in each study. In addition, spectra were acquired using the same pulse sequence preceded by an 8 -ms hyperbolic secant inversion pulse with a delay of $1.01 \mathrm{~s}$ before the excitation. The delay of this IR pulse sequence was chosen to suppress the signals from metabolites with long $\mathrm{T} 1$ relaxation times and leave signals that arise predominantly from macromolecules and exchangeable protons that have relatively short $\mathrm{T} 1$ relaxation times (19).

Processing and quantitation. Spectra from the data acquired using the first pulse sequence were obtained by zero filling from 4000 to 32000 points, Gaussian or exponential multiplication, and convolution difference of the time domain data. Baseline correction was performed on each spectrum before measurement of the peak height. The ratio of the peak height at $\sim 7.3 \mathrm{ppm}$ (Phe) and $7.85 \mathrm{ppm}$ (amide protons of $\mathrm{N}$ acetylaspartate) was compared in both control subjects and subjects with PKU. If the peak height ratio was at least 30\% greater than that observed in controls, the study was deemed positive for the presence of Phe.

For quantitation of data obtained from the second pulse sequence, the time domain data were zero filled from 2000 to 32000 points and filtered with a Lorentzian to Gaussian function for optimization of signal-to-noise ratio, which resulted in a 3-Hz line broadening after Fourier transformation. Difference spectra were obtained by subtraction of the time domain data obtained with and without the preceding IR pulse and then processed as just described. Zero and first-order polynomial baseline correction (10 to $6.0 \mathrm{ppm}$ for Phe and 4.0 to $0 \mathrm{ppm}$ for $\mathrm{Cr}$ ) of the spectra was performed before they were used to quantitate the Phe concentrations by measuring the peak height of the resonance at $7.34 \mathrm{ppm}$ and comparing it to the height of the $\mathrm{Cr}$ resonance at $3.04 \mathrm{ppm}$. In the spectra obtained in vivo, the half-height line width of the $\mathrm{Cr}$ resonance was from 7 to 10 $\mathrm{Hz}$ after line broadening. The Phe concentration was calculated 
using the following formula:

$$
[\mathrm{Phe}]=\left(\mathrm{Phe}^{*} / \mathrm{Cr}^{*}\right) \times \mathrm{CF}_{\mathrm{P}} \times \mathrm{CF}_{\mathrm{L}} \times[\mathrm{Cr}],
$$

where $\mathrm{Phe}^{*}$ and $\mathrm{Cr}^{*}$ are the peak heights of the $\mathrm{Phe}$ and $\mathrm{Cr}$ signals. $\mathrm{CF}_{\mathrm{P}}$ is the correction factor for the semiselective pulse and differences in line widths determined from performing the same localized ${ }^{1} \mathrm{H}$ NMR experiment on a phantom of $10 \mathrm{mM}$ Phe and $10 \mathrm{mM} \mathrm{Cr}$ in phosphate buffer $(150 \mathrm{mEq} \mathrm{KCl}$ / $\mathrm{K}_{2} \mathrm{HPO}_{4} ; \mathrm{pH}=7.1$; temperature $=37^{\circ} \mathrm{C}$ ). The line width of $\mathrm{Cr}$ in the phantom was line broadened to match the in vivo data for each subject. $\mathrm{CF}_{\mathrm{L}}$ is the correction factor for the differences in line width of the $\mathrm{Phe}$ and $\mathrm{Cr}$ signals observed in vivo. This was determined from the difference spectra obtained from the subject with the most intense Phe resonance and a control subject. The [Cr] is the in vivo concentration of $\mathrm{Cr}$ in the occipital lobe, which was assumed to be $10 \mathrm{mM}$ from in vitro studies performed on human cortical tissue (20). The peak height ratios of NA/Cr and NA/TMA resonances were also measured in the difference spectra obtained in both controls and subjects with PKU. These were compared using $t$ test.

\section{RESULTS}

Figure 1 shows a T1-weighted MRI, the ${ }^{1} \mathrm{H}$ spectrum of a normal subject, the $\mathrm{T} 1$-weighted ${ }^{1} \mathrm{H}$ spectrum showing the signals that arise predominantly from macromolecules, and the difference spectrum using the second pulse sequence. Note the

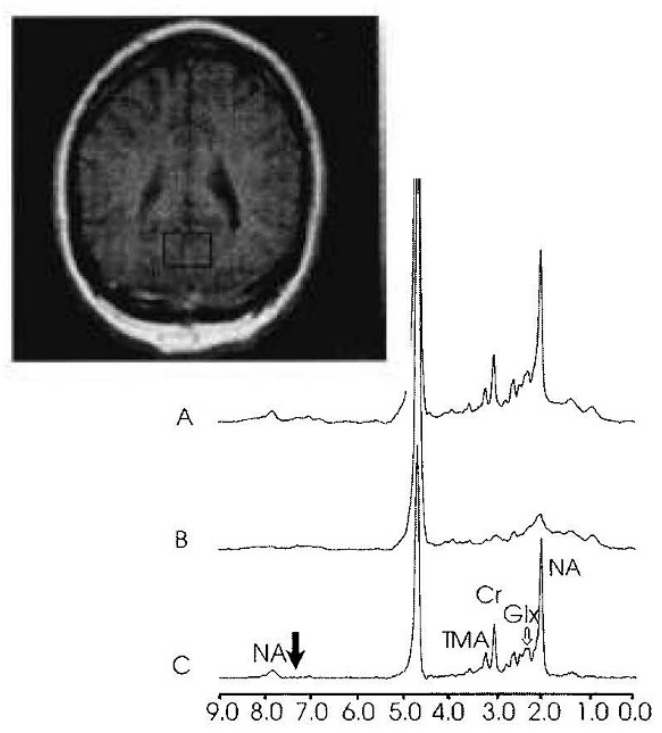

PPM

Figure 1. Localized proton NMR spectra and image of control subject. Localized proton spectra from the occipital region of a control subject from the area depicted in T1-weighted MRI (inset). Spectrum A was acquired over a 6-min period. Spectrum B is a T1-weighted ${ }^{1} \mathrm{H}$ spectrum showing signals from metabolites with short $\mathrm{T} 1$ relaxation times, which are primarily macromolecules. Spectrum C is the result of subtracting spectrum B from spectrum A and shows the proton signals of compounds with relatively longer $\mathrm{T} 1$ relaxation times. The labeled metabolites include NA, glutamate and glutamine $(G l x)$, total $\mathrm{Cr}, \mathrm{TMA}$, and the amide protons of $\mathrm{N}$-acetylaspartate. The residual water signal is observed at $\sim 4.7 \mathrm{ppm}$, and the downfield region of the spectrum where the signal from the aromatic protons of Phe are observed is depicted by the arrow. relative intensities of the signals from the other metabolites versus the signals in the downfield region of the spectra. Figure 2 depicts ${ }^{1} \mathrm{H}$ spectra of the downfield regions of subjects with PKU, controls, and a phantom of Phe using both pulse sequences. Both the control spectra and spectra from subjects with PKU show signals from an unassigned resonance at 7.05 ppm, a peak at $\sim 7.3 \mathrm{ppm}$, and the amide ${ }^{1} \mathrm{H}$ of $\mathrm{N}$ acetylaspartate at $7.85 \mathrm{ppm}$. In the subjects with PKU, the height of the peak at $\sim 7.3 \mathrm{ppm}$ is greater and shifted further downfield compared with that in normal subjects (7.36 for PKU subjects versus 7.30 for normal subjects). The T1weighted spectrum demonstrates that there is a significant

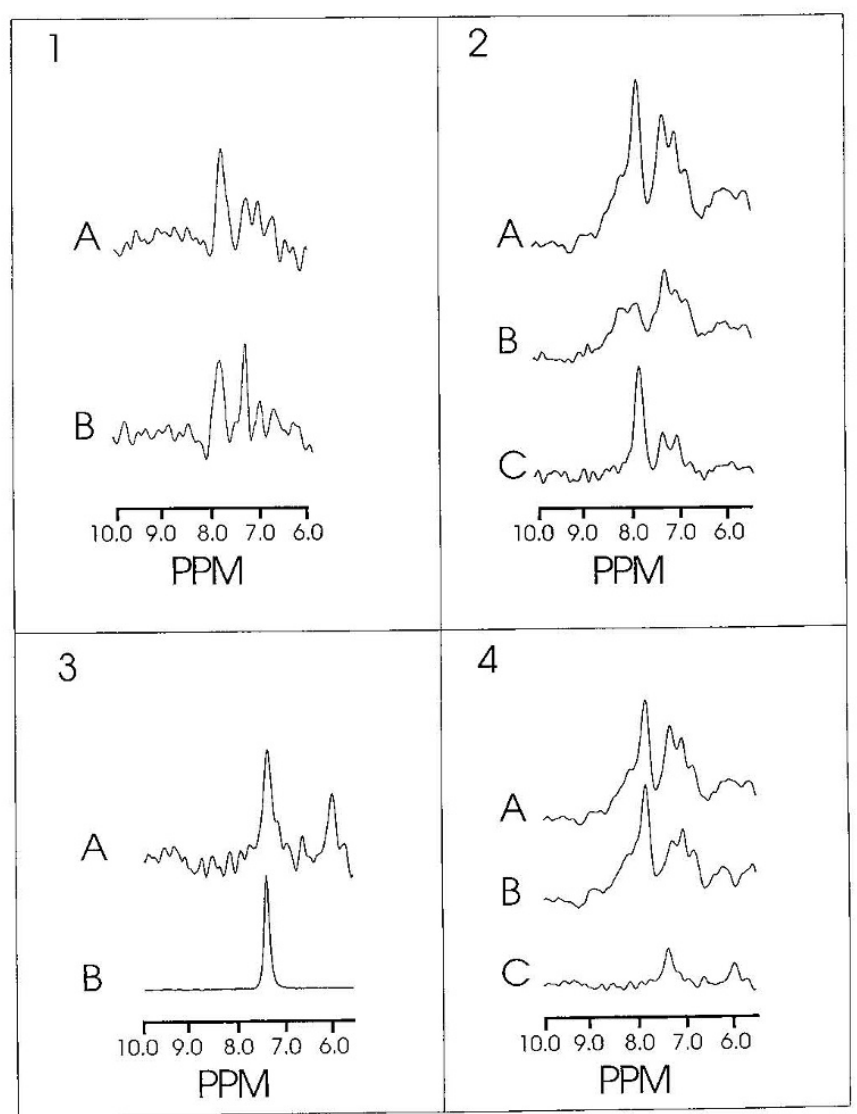

Figure 2. Localized proton spectra of downfield region in control and PKU subjects. A series of ${ }^{1} \mathrm{H}$ spectra from the downfield region magnified 16 to 20 times the spectra shown in Figure 1 is depicted in four panels. Panel 1 shows spectra using the first pulse sequence described in the text. Spectrum $\mathrm{A}$ is from a control subject and spectrum B is from a subject with PKU whose serum Phe was $1.83 \mathrm{mM}$ at the time of the study. Panel 2 shows three proton spectra obtained using the second pulse sequence described in text and is from a subject with PKU whose serum Phe was $1.96 \mathrm{mM}$. Spectrum A shows proton signals from all compounds in this region, and B shows peaks from compounds with short $\mathrm{T} 1$ relaxation times. Spectrum $\mathrm{C}$ is the difference spectrum from $\mathrm{A}$ and $\mathrm{B}$ and shows the peak of the amide protons of $\mathrm{N}$-acetyl aspartate at 7.85 $\mathrm{ppm}$, an as yet unidentified signal at $7.05 \mathrm{ppm}$, and the peak from the aromatic protons of Phe at $7.34 \mathrm{ppm}$. Panel 3 shows the in vivo difference spectrum from a control and a subject with PKU in $A$ and the proton spectrum from a solution of Phe and $\mathrm{Cr}(\mathrm{B})$ obtained using the same NMR experiment. The spectrum in $\mathrm{B}$ was obtained by line broadening the signal so that the line width of the $\mathrm{Cr}$ signal in the phantom solution matched that of the in vivo ${ }^{1} \mathrm{H}$ difference spectrum from the subject with PKU. Panel 4 shows ${ }^{1} \mathrm{H}$ spectra from a subject with PKU whose serum Phe was $1.96 \mathrm{mM}$ in A and a control subject in B. Spectrum C is the difference (A minus B) and demonstrates the residual peak from the aromatic protons of Phe at $7.34 \mathrm{ppm}$. 
contribution of signals from compounds with short longitudinal relaxation times in this region of the spectrum. Subtraction of the T1-weighted spectrum eliminated most of the intensity at $7.3 \mathrm{ppm}$ in the control subjects. In contrast, in subjects with PKU a resonance was observed at $7.34 \mathrm{ppm}$. The difference spectrum from a subject with PKU and a normal subject shows a signal that has a chemical shift similar to that of the spectrum from the phantom with $\mathrm{Phe}$ and $\mathrm{Cr}$. The in vivo Phe resonance was broadened by $\sim 7 \mathrm{~Hz}$.

Due to the presence of underlying short $\mathrm{T} 1$ signals and greater variation in shimming and water suppression in the initial studies, quantitation of the Phe signal was performed only with data obtained using the second pulse sequence. The serum Phe concentrations of the subjects with PKU ranged from 10.8 to $54.8 \mathrm{mg} / \mathrm{dL}(0.7$ to $3.3 \mathrm{mM})$ at the time the spectra were acquired. Table 1 shows the age of the subjects and blood levels of Phe in the PKU subjects at the time of the study and the measured brain concentrations of Phe. The brain Phe concentrations were 0.48 to $0.78 \mathrm{mM}$ in the subjects with PKU and $0.21 \pm 0.02 \mathrm{mM}$ in controls, which may represent overlap from neighboring peaks. There was a poor correlation between blood and brain concentrations in this study.

In addition to measurement of Phe, ${ }^{1} \mathrm{H}$ NMR spectroscopy permits assessment of several other metabolites including NA, glutamate, glutamine, $\mathrm{Cr}$, and trimethylamines in both subjects and controls. The peak height ratios of NA/Cr, NA/TMA, and $\mathrm{Cr} / \mathrm{TMA}$ were $2.67 \pm 0.07,5.1 \pm 0.3$, and $1.9 \pm 0.2$ for subjects with PKU and 2.6 $\pm 0.1,5.4 \pm 0.5$, and $2.1 \pm 0.1$ for controls. We observed no statistically significant difference between the NA/Cr, NA/TMA, and Cr/TMA ratios in spectra from controls and subjects with PKU. The concentrations of the other amino acids were not quantitated because the pulse sequence was not optimized to measure these amino acids as we have previously described (21); however, qualitatively we observed no differences between spectra from control and PKU subjects. The inversion recovery or T1-weighted spectrum permits assessment of changes in macromolecules. Again we observed no qualitative differences in the spectra of subjects with PKU.

Table 1. Age, serum Phe levels at time of study, and brain concentrations of Phe in subjects with $P K U$

\begin{tabular}{|c|c|c|c|c|c|}
\hline \multirow[b]{2}{*}{ Study } & \multirow[b]{2}{*}{ Subject } & \multirow{2}{*}{$\begin{array}{l}\text { Age } \\
(y)\end{array}$} & \multicolumn{2}{|c|}{ Plasma [Phe] } & \multirow{2}{*}{$\begin{array}{c}\text { Brain [Phe] } \\
(\mathrm{mM})\end{array}$} \\
\hline & & & $\mathrm{mg} / \mathrm{dL}$ & $\mathrm{mM}$ & \\
\hline 1 & A & 22 & 46.40 & 2.81 & + \\
\hline 2 & B & 18 & 54.80 & 3.32 & + \\
\hline 3 & C & 22 & 47.60 & 2.88 & + \\
\hline 4 & D & 22 & 52.80 & 3.20 & + \\
\hline 5 & D & 23 & 36.00 & 2.18 & + \\
\hline 6 & $\mathrm{E}$ & 18 & 22.90 & 1.39 & + \\
\hline 7 & A & 23 & 30.30 & 1.83 & $t$ \\
\hline 8 & $\mathrm{~F}$ & 13 & 10.80 & 0.65 & 0.48 \\
\hline 9 & G & 26 & 17.60 & 1.07 & 0.67 \\
\hline 10 & A & 28 & 32.40 & 1.96 & 0.53 \\
\hline 11 & $\mathrm{H}$ & 14 & 25.60 & 1.55 & 0.78 \\
\hline 12 & A & 28 & 38.00 & 2.30 & 0.74 \\
\hline
\end{tabular}

The brain concentration of Phe in controls $(n=5)$ was $0.21 \pm 0.02 \mathrm{mM}$. The + indicates that the Phe signal at $\sim 7.35$ ppm was observed in these subjects.

\section{DISCUSSION}

NMR spectroscopy has been used to investigate human cerebral metabolism for more than a decade. For technical reasons, early human studies were restricted to investigations using ${ }^{31} \mathrm{P}$ NMR techniques to measure high-energy phosphates in brain and muscle (22). The ${ }^{1} \mathrm{H}$ nucleus provides the greatest sensitivity of all NMR spectroscopic techniques and permits measurement of metabolites with concentrations on the order of $0.1 \mu \mathrm{mol} / \mathrm{g}$, which include lactate, NA, Cr, TMA, and myo-inositol. Use of short echo times has permitted measurement of glucose (23), glutamate (21), glutamine (24), $\gamma$-aminobutyric acid (18), and taurine (25), which are present at high enough concentrations in the normal human brain to be measured by ${ }^{1} \mathrm{H}$ NMR. At the magnetic fields available for most clinical studies, the resolution of the ${ }^{1} \mathrm{H}$ spectrum is restricted and makes accurate quantitation of these metabolites difficult. In addition, the signals of the metabolites are also superimposed on ${ }^{1} \mathrm{H}$ signals of macromolecules (19). In some cases, these problems have been overcome by development of editing techniques to permit measurement of specific metabolites that have certain ${ }^{1} \mathrm{H}$ NMR spectroscopic characteristics $(18,26)$. In many neuropathologic disorders, particularly neurometabolic and neurodegenerative diseases, the concentrations of many metabolites increase to a level that can be measured by ${ }^{1} \mathrm{H}$ NMR techniques. Many of the leukodystrophies such as Canavan's disease, adrenoleukodystrophy, and metachromatic leukodystrophy have been shown to have characteristic changes in metabolites (27-29). Disorders of intermediary and energy metabolism elevate tissue levels of lactate into a range that can be readily measured with ${ }^{1} \mathrm{H}$ NMR spectroscopy (30). The aminoacidopathies and hyperammonemias cause elevations in cerebral concentrations of several amino acids that are measurable by this technique (24). An early animal study in rats with histidinemia suggested that ${ }^{1} \mathrm{H}$ NMR spectroscopy could be used to study this and other aminoacidopathies including PKU (31). A study in rabbits with chemically induced hyperphenylalaninemia demonstrated that both Phe and metabolites of Phe were measurable in the brain (15).

This study demonstrates that ${ }^{1} \mathrm{H}$ NMR spectroscopy can be used to measure Phe in localized brain regions of subjects with PKU at the concentrations expected in untreated individuals with this metabolic disorder. The brain concentrations measured in vivo in this study are comparable to those measured by in vitro methods in postmortem brain specimens of untreated PKU patients $(32,33)$. A significant finding in our study is that the brain concentration is significantly lower than the blood concentration, and there is a poor correlation between the blood and brain concentrations.

Three other ${ }^{1} \mathrm{H}$ NMR studies have been performed on subjects with PKU $(13,34,35)$. Two studies used a spin echo pulse sequence where the echo time was too long to permit observation of Phe, which has a short apparent spin-spin relaxation time secondary to the complex $J$ modulation of this multiplet $(13,34)$. The latter study suggested that there were alterations in ratios of $\mathrm{NA} / \mathrm{Cho}$ and $\mathrm{Cho} / \mathrm{Cr}$ in subjects with PKU compared with controls in ${ }^{1} \mathrm{H}$ spectra obtained from a $2-\mathrm{cm}^{3}$ volume in the right posterior hemisphere. Another study 
using a stimulated echo pulse sequence with a short echo time ( $\mathrm{TE}=20 \mathrm{~ms}$ ) and a volume coil was also unable to locate Phe or any of the metabolites downfield of water observed in controls (35). This study agreed with our results in that it reported no changes in the ratios of $\mathrm{Cho} / \mathrm{Cr}, \mathrm{NA} / \mathrm{Cr}$, or NA/Cho between subjects with PKU and controls. The neurobiologic significance of the findings in these studies remains to be explained. The possibility that the changes were due to normal intrasubject variation needs to be explored. Despite the use of a short echo time, Phe was not observed in vivo in the latter study. This may be explained by the use of a volume coil, the lower field strength of the magnet, and the stimulated echo pulse sequence, all of which decrease sensitivity of the NMR study. A recent abstract from Kreis et al. (36) has reported similar findings to the present study.

The Phe resonance at $7.3 \mathrm{ppm}$ in the ${ }^{1} \mathrm{H}$ spectrum includes signals from all five aromatic protons of Phe molecules. The technique cannot distinguish the signal from metabolites of Phe in which the chemical environment of the aromatic protons is not significantly altered. The signal of Phe molecules that are bound to proteins or other macromolecules is reduced in intensity (37). Phenylacetate, phenylpyruvate, and phenyllactate are Phe metabolites that have been observed in the brains of animal models of PKU. The signals from the aromatic protons in these metabolites are shifted significantly away from the 7.3-ppm region. Any alteration of the aromatic ring will also cause a significant shift of the signal. Addition of other chemical moieties on the amino group may not result in a shift of the aromatic proton signal. $\gamma$-Glutamylphenylalanine has been observed in the urine of subjects with PKU (38). This compound may also be in the brain, inasmuch as there is a similar enzyme system in the endothelium of the blood brain barrier which is involved with transport of amino acids and other metabolites. This compound may contribute to the ${ }^{1} \mathrm{H}$ NMR signal observed in vivo and could account for the greater observed in vivo line width. In both PKU and control subjects, a large fraction of the 7.3 signal was from short $\mathrm{T} 1$ compounds. The presence of these resonances in this region is consistent with our previous demonstration of large short T1 resonances in the upfield region of the spectrum (19). Quantitation of Phe by subtraction of the IR spectrum was compared with difference spectra from PKU and control subjects and found to give similar results. The IR difference method has the advantages of separating changes in Phe from potential changes in the short $\mathrm{T} 1$ background as well as not depending on the assumption that $\mathrm{Cr}$ or some other endogenous compound is the same in both groups.

${ }^{1} \mathrm{H}$ NMR spectroscopy can be used to determine the correlation of cerebral Phe levels with neuropsychologic dysfunction and neurophysiologic measurements (39), which is especially important if blood and brain levels of Phe correlate poorly, as we observed. Because multiple serial measurements can be made noninvasively, the technique can be used to monitor the efficacy of and compliance with therapeutic interventions. This will be particularly useful in monitoring the efficacy of alternative dietary therapies and somatic cell gene therapy (40). The transport of Phe into the nervous system can also be measured. ${ }^{1} \mathrm{H}$ NMR studies on transport of this amino acid into the nervous system will permit investigations of how an individual adapts to chronic hyperphenylalaninemia and determine whether certain individuals may alter Phe transport across the blood-brain barrier.

This study demonstrates that ${ }^{1} \mathrm{H}$ NMR spectroscopy can now measure cerebral metabolites in the downfield region in ${ }^{1} \mathrm{H}$ spectra from the human brain. This complements the previously demonstrated capability of ${ }^{1} \mathrm{H}$ NMR to quantitatively measure other compounds. A measure of signals from macromolecules can also be obtained using T1-weighted spectroscopic methods (19). This technique will be particularly useful for the investigations of neurometabolic and neurodegenerative diseases in which the biochemical and molecular changes diffusely affect the CNS. Another major advantage of the NMR technique is that it can be applied to investigations of cerebral metabolism in infants and children.

\section{REFERENCES}

1. Scriver CR, Kaufman S, Woo SLC 1988 Mendelian hyperphenylalanemia. Annu Rev Genet 22:301-321

2. Scriver CR 1991 Phenylketonuria: genotypes and phenotypes. N Engl J Med 324:1280-1281

3. Okano Y, Eisensmith RC, Guttler F, Lichter-Konecki U, Konecki DS, Trefz FK, Dasovich M, Wang T, Henriksen K, Lou H 1991 Molecular basis of phenotypic heterogeneity in phenylketonuria. N Engl J Med 324:1232-1238

4. Trefz FK, Burgard P, Konig T, Goebel-Schreiner B, Lichter-Konecki U, Konecki D, Schmidt E, Schmidt H, Bickel H 1993 Genotype-phenotype correlations in phenylketonuria. Clin Chim Acta 217:15-21

5. Krause W, Halminski M, McDonald L, Dembure P, Salvo R, Freides D, Elsas L 1985 Biochemical and neuropsychological effects of elevated plasma phenylalanine in patients with treated phenylketonuria. A model for the study of phenylalanine and brain function in man. J Clin Invest 75:40-48

6. Partington MW 1978 Long term studies of untreated phenylketonuria II: the plasma phenylalanine level. Neuropediatrics 9:255-267

7. Kaufman S 1989 An evaluation of the possible neurotoxicity of metabolites of phenyalanine. J Pediatr 114:895-900

8. Clemens PC, Schunemann MH, Kohlschutter A, Hoffman GF 1990 Phenylalanine metabolites in phenylketonuria. J Pediatr 116:665-666

9. Seashore MR, Friedman E, Novelly RA, Bapat V 1985 Loss of intellectual function in children with phenylketonuria after relaxation of dietary phenylalanine restriction. Pediatrics $75: 226-232$

10. Waisbren SE, Mahon BE, Schnell RR, Levy HL 1987 Predictors of intelligence quotient and intelligence quotient change in persons treated for phenylketonuria early in life. Pediatrics 79:351-355

11. Thompson AJ, Tillotson S, Smith I, Kendall B, Moore SG, Brenton DP 1993 Brain MRI changes in phenylketonuria. Associations with dietary status. Brain 116:811821

12. Pearsen KD, Gean-Marton AD, Levy HL, Davis KR 1990 Phenylketonuria: MR imaging of the brain with clinical correlation. Radiology 177:437-440

13. Bick U, Ullrich K, Stober U, Moller H, Schuierer G, Ludolph AC, Oberwittler C, Weglage J, Wendel U 1993 White matter abnormalities in patients with treated hyperphenylalaninaemia: magnetic resonance relaxometry and proton spectroscopy findings. Eur $\mathrm{J}$ Pediatr 152:1012-1020

14. Cockburn F, Barwell BE, Brenton DP 1993 Recommendations on the dietary management of phenylketonuria. Arch Dis Child 68:426-427

15. Avison MJ, Herschkowitz N, Novotny EJ, Petroff OA, Rothman DL, Colombo JP, Bachmann C, Shulman RG, Prichard JW 1990 Proton NMR observation of phenylalanine and an aromatic metabolite in the rabbit brain in vivo. Pediatr Res 27:566-570

16. Ordridge RJ, Connelly A, Lohman JAB 1986 Image-selected in vivo spectroscopy (ISIS). A new technique for spatially selective NMR spectroscopy. J Magn Reson 66:283-294

17. Gruetter R 1993 Automatic, localized in vivo adjustment of all first- and second-order shim coils. Magn Reson Med 29:804-811

18. Rothman DL, Petroff OA, Behar KL, Mattson RH 1993 Localized ${ }^{1} \mathrm{H}$ NMR measurements of gamma-aminobutyric acid in human brain in vivo. Proc Natl Acad Sci USA 90:5662-5666

19. Behar KL, Rothman DL, Spencer DD, Petroff OAC 1994 Analysis of macromolecule resonances in ${ }^{1} \mathrm{H}$ NMR spectra of human brain. Magn Reson Med 32:294-302

20. Petroff OA, Spencer DD, Alger JR, Prichard JW 1987 High-field proton magnetic resonance spectroscopy of human cerebrum obtained during surgery for epilepsy. Neurology 39:1197-1.202

21. Rothman DL, Hanstock CC, Petroff OA, Novotny EJ, Prichard JW, Shulman RG 1992 Localized ${ }^{1} \mathrm{H}$ NMR spectra of glutamate in the human brain. Magn Reson Med 25:94-106

22. Griffiths RD, Edwards RHT 1987 Magnetic resonance spectroscopy in the recognition of metabolic disease. J Inherit Metab Dis 10:147-158 
23. Chen W, Novotny EJ, Zhu XH, Rothman DL, Shulman RG 1993 Localized ${ }^{1} \mathrm{H}$ NMR measurement of glucose consumption in the human brain during visual stimulation. Proc Natl Acad Sci USA 90:9896-9900

24. Kreis R, Ross BD, Farrow NA, Ackerman Z 1992 Metabolic disorders of the brain in chronic hepatic encephalopathy detected with H-1 MR spectroscopy. Radiology 182:19-27

25. Huppi PS, Posse S, Lazeyras F, Burri R, Bossi E, Herschkowitz N 1991 Magnetic resonance in preterm and term newborns: ${ }^{1} \mathrm{H}$-spectroscopy in developing human brain. Pediatr Res 30:574-578

26. Rothman DL, Behar KL, Hetherington HP, Shulman RG 1984 Homonuclear ${ }^{1} \mathrm{H}$ double-resonance difference spectroscopy of the rat brain in vivo. Proc Natl Acad Sci USA 81:6330-6334

27. Marks HG, Caro PA, Wang ZY, Detre JA, Bogdan AR, Gusnard DA, Zimmerman RA 1991 Use of computed tomography, magnetic resonance imaging, and localized ${ }^{1} \mathrm{H}$ magnetic resonance spectroscopy in Canavan's disease: a case report. Ann Neurol 30:106-110

28. Kruse B, Hanefeld F, Christen HJ, Bruhn H, Michaelis T, Hanicke W, Frahm J 1993 Alterations of brain metabolites in metachromatic leukodystrophy as detected by localized proton magnetic resonance spectroscopy in vivo. J Neurol 241:68-74

29. Grodd W, Krageloh-Mann I, Klose U, Sauter R 1991 Metabolic and destructive brain disorders in children: findings with localized proton MR spectroscopy. Radiology 181:173-181

30. Krageloh-Mann I, Grodd W, Schoning M, Marquard K, Nagele T, Ruitenbeek W 1993 Proton spectroscopy in five patients with Leigh's disease and mitochondrial enzyme deficiency. Dev Med Child Neurol 35:769-776

31. Gadian DG, Proctor E, Williams SR, Cady EB, Gardiner RM 1986 Neurometabolic effects of an inborn error of amino acid metabolism demonstrated in vivo by ${ }^{1} \mathrm{H} N M R$. Magn Reson Med 3:150-156
32. McKean CM, Peterson NA 1970 Glutamine in the phenylketonuric central nervous system. N Engl J Med 283:1364-1367

33. Adriaenssens K, Allen RJ, Lowenthal A, Mardens X, Tourtellotte WW 1969 Brain and cerebrospinal fluid free amino acids in phenylketonuria. J Genet Hum 17:223 230

34. Hajek M, Hejcmanova L, Pradny J 1993 Proton in vivo spectroscopy of patients with hyperphenylalaninaemia. Neuropediatrics 24:111-112

35. Johannik K, Van Hecke P, Francois B, Marchal G, Smet MH, Jaeken J, Breysem L, Wilms G, Baert AL 1994 Localized brain proton NMR spectroscopy in young adult phenylketonuria patients. Magn Reson Med 31:53-57

36. Kreis R, Pietz J, Penzien J, Herschkowitz N, Boesch C 1994 Unequivocal identification and quantitation of phenylalanine in the brain of patients with phenylketonuria by means of localized in vivo ${ }^{1} \mathrm{H}$ MRS. Proceedings of the Society of Magnetic Resonance 1:308(abstr)

37. Nicholson JK, Gartland KPR $1989{ }^{1} \mathrm{H}$ NMR studies on protein binding of histidine, tyrosine and phenylalanine in blood plasma. NMR Biomed 2:77-82

38. Kamerling JP, Aarsen GJ, Duran M, de Bree PK, van Sprang FJ, Wadman SK 1980 Chromatographic determination and mass spectrometric identification of gammaglutamylphenylalanine, a urinary constituent in phenylketonuria. Clin Chim Acta 102:137-145

39. Pietz J, Schmidt E, Matthis P, Kobialka B, Kutscha A, de Sonneville L 1990 EEGs in phenylketonuria. I: Follow-up to adulthood; II: Short-term diet-related changes in EEGs and cognitive function. Dev Med Child Neurol 35:54-64

40. Cristiano RJ, Smith LC, Woo SL 1993 Hepatic gene therapy: adenovirus enhancement of receptor-mediated gene delivery and expression in primary hepatocytes. Proc Natl Acad Sci USA 90:2122-2126 